\title{
Do present damage and health perception in patients with systemic lupus erythematosus predict extent of future damage?: a prospective study
}

\author{
Thomas Stoll, Nurhan Sutcliffe, Richard Klaghofer, David A Isenberg
}

\begin{abstract}
Objective-To study whether either initial damage, disease activity, disease duration, age, a drug score, or health status would predict an increase in damage in patients with systemic lupus erythematosus (SLE) within the next three years.

Methods-A three year prospective longitudinal study of a cohort of 141 consecutive patients with SLE attending a specialist lupus outpatient clinic from their first assessment between July 1994 and February 1995. Disease activity was assessed using the BILAG system, health status by the Medical Outcome Survey Short Form 20 with an extra question about fatigue (SF-20+), and damage by the SLICC/ACR Damage Index (SDI). Damage was reassessed three years later. Statistical analysis was carried out using logistic regression analysis (logXact).

Results-133 female and 8 male patients with SLE (97 white subjects, 16 AfroCaribbeans, 22 Asians, and 6 others) were included. Their mean (SD) age at inclusion was 41.1 (12.5) years and their disease duration 10.2 (6.3) years. The mean measures at inclusion were: total BILAG 5.2 (range 0-17), total SDI $1.2(0-7)$, drug score 1.2 (0-3); SF-20+: physical 58 (0-100), role $54(0-100)$, social functioning $71(0-100)$, mental health 64 (16-100), health perception $44(0-100)$, pain $53(0-100)$, fatigue 59 $(0-100)$. Four patients were lost to follow up because they had moved. At three years in 33 patients the total SDI had increased to a mean of $1.5(0-7)(n=130)$. Moreover, seven patients had the maximum damage as they had died during the follow up period. The only variables with an independent and significant contribution in predicting damage at three years were the total damage score $($ odds ratio $(O R)=1.46 ; 95 \%$ CI 1.04 to $2.05)$, and health perception $(O R=0.96 ; 95 \%$ CI 0.93 to 0.99 ) at inclusion.

Conclusions-Of all the variables at inclusion only the total damage score and SF-20+: health perception, significantly predicted an increase in damage, for patients with SLE, three years later.

(Ann Rheum Dis 2000;59:832-835)
\end{abstract}

Systemic lupus erythematosus (SLE) is an autoimmune rheumatic disease characterised by periods of active disease and remission with a heterogeneous clinical pattern, as organ/ system involvement in different patients can be extremely variable in extent and severity. Episodes of active disease can be life threatening but are usually controlled by immunosuppressive treatment. Although periods of active disease are associated with reversible impairment, these episodes may also cause permanent damage. Reversible impairment has been shown to affect health status assessed by patients with SLE more than damage. ${ }^{1}$ Whereas instruments to measure disease activity have been developed and used for 30 years, ${ }^{2}$ the major development in the assessment of damage followed a meeting in Boston (1991) of the Systemic Lupus International Collaborative Clinics (SLICC) group. ${ }^{3}$ The SLICC Damage Index was shown to be valid at the same time (March 1996) in Canada/USA and in Europe. ${ }^{45}$ Since its acceptance and approval by the American College of Rheumatology it has been named the SLICC/ACR Damage Index (SDI) and is now used widely. Although many groups have assessed patients with SLE by SDI in cross sectional studies, sparse data are available on its longitudinal and long term use in real patients. Therefore, we followed up our SLE cohort ${ }^{6}$ over three years and reassessed damage. The present investigation aimed at studying whether either initial damage, disease activity, disease duration, age, a drug score, or health status would predict an increase in damage within the next three years. We suggested that according to published reports ${ }^{5}$ initial damage would predict future damage. Moreover, we expected that if one section of the patient administered health status questionnaire Short Form 20 with an extra question about fatigue (SF-20+) could predict future damage, it might be the most general health status measure-that is, health perception, as SLE is a systemic disease.

\section{Methods}

STUDY DESIGN

A prospective longitudinal study of a cohort of 141 consecutive patients with SLE attending a specialist lupus outpatient clinic and having their first assessment between July 1994 and 
Table 1 Disease characteristics of the 141 patients with systemic lupus erythematosus at the first assessment and, in square brackets, damage scores three years later $(n=130)$

\begin{tabular}{lll}
\hline SDI/BILAGt, SF-20+t, drug scores & Mean (SD) & Median; range \\
\hline Total damage & $1.2(1.5)[1.5(1.6)]$ & $1 ; 0-7[1 ; 0-7]$ \\
Neuropsychiatric damage & $0.21(0.49)[0.25(0.61)]$ & $0 ; 0-2[0 ; 0-3]$ \\
Renal damage & $0.31(0.74)[0.32(0.72)]$ & $0 ; 0-3[0 ; 0-3]$ \\
Musculoskeletal damage & $0.27(0.56)[0.34(0.71)]$ & $0 ; 0-3[0 ; 0-3]$ \\
BILAG total score & $5.2(3.8)$ & $4 ; 0-17$ \\
General BILAG & $1(1.1)$ & $1 ; 0-9$ \\
Mucocutaneous BILAG & $0.7(1.2)$ & $0 ; 0-9$ \\
Neurological BILAG & $0.3(0.9)$ & $0 ; 0-9$ \\
Musculoskeletal BILAG & $1.2(2)$ & $1 ; 0-9$ \\
Cardiovascular/respiratory BILAG & $0.3(0.6)$ & $0 ; 0-3$ \\
Vasculitis BILAG & $0.4(0.7)$ & $0 ; 0-3$ \\
Renal BILAG & $0.3(0.9)$ & $0 ; 0-3$ \\
Haematological BILAG & $1(1.2)$ & $0 ; 0-9$ \\
SF-20+: physical functioning & $58(34)$ & $67 ; 0-100$ \\
SF-20+: role functioning & $54(42)$ & $50 ; 0-100$ \\
SF-20+: social functioning & $71(30)$ & $80 ; 0-100$ \\
SF-20+: mental health & $64(21)$ & $68 ; 16-100$ \\
SF-20+: health perception & $44(25)$ & $42 ; 0-100$ \\
SF-20+: pain & $53(35)$ & $50 ; 0-100$ \\
SF-20+: fatigue & $59(29)$ & $70 ; 0-100$ \\
Drug score & $1.2(1.3)$ & $1 ; 0-3$ \\
\hline
\end{tabular}

Organ damage item only given if mean score at inclusion $\geqslant 0.1$ (and at three years $>0.12$ ). Lower scores always mean better health except for the SF-20+ scores marked with *

+ SDI $=$ SLICC/ACR Damage Index; SF-20+ = Short Form 20 with an extra question about fatigue.

February $1995^{6}$ was undertaken. Damage was reassessed three years later at a visit of the patients to the same outpatient clinic.

DATA COLLECTION

Each patient with SLE was first assessed at an outpatient consultation between July 1994 and February 1995. The date at which a patient fulfilled four of the revised classification criteria established by the American College of Rheumatology ${ }^{7}$ was recorded as the date of diagnosis. Disease duration (years) was calculated by subtracting the date of first assessment from the date of diagnosis. Disease activity was recorded using the BILAG system. ${ }^{8}{ }^{9}$ Damage was also assessed three years later using the SDI. ${ }^{4510}$ Shortly before or after the consultation, on the same day, patients completed the SF-20+. ${ }^{112}$ A drug score was determined by the doctor. ${ }^{13}$

\section{MEASURES OF ASSESSMENT}

Activity

BILAG measures SLE disease activity in eight organ based components (general, mucocutaneous, neurological, musculoskeletal, cardiovascular/respiratory, vaculitis, renal, and haematological) and is based upon the principle of the doctor's intention to treat. ${ }^{8}$ To obtain a global score, BILAG component scores can be assigned numerical values: $A=9$ (most active disease), $\mathrm{B}=3$ (intermediate activity), $\mathrm{C}=1$ (mild and stable disease activity), $\mathrm{D}=0$ (inactive disease), and $\mathrm{E}=0$ (no activity ever), resulting in a potential summed range from 0 to 72 points. This numeric score has been shown to be valid. ${ }^{9}$

\section{SLICC/ACR Damage Index (SDI)}

Damage - that is, irreversible impairment since onset of SLE, is usually defined as a clinical feature that has to be continuously present for at least six months to score. The components have been reported in detail elsewhere. ${ }^{4}{ }^{10}$ Briefly, damage is defined for 12 organ systems: ocular (range 0-2), neuropsychiatric
$(0-6)$, renal $(0-3)$, pulmonary $(0-5)$, cardiovascular $(0-6)$, peripheral vascular $(0-5)$, gastrointestinal (0-6), musculoskeletal (0-7), skin $(0-3)$, endocrine (diabetes) (0-1), gonadal $(0-1)$, and malignancies (0-2). Damage over time can only be stable or increase, theoretically to a maximum of 47 points.

MOS Short Form 20 with an additional question for fatigue (SF-20+)

The 20 questions of the SF-20 comprise six sections: physical, role, social functioning, mental health, health perception, and pain. As explained elsewhere a further question about fatigue was added, and we multiplied the numeric rating scale $(0-10)$ by $10 .{ }^{6}$ For most of the scale scores higher scores mean better health (range 0-100). Only the scores for fatigue and pain have the opposite polarity so that 0 means not fatigued at all or no pain. ${ }^{6} 1112$

\section{Drug score}

Drug score ranged from 0 to 3 ( $0=$ neither prednisone nor immunosuppressant drugs, $1=$ prednisone $1-20 \mathrm{mg} / \mathrm{d}, 2=$ prednisone $>20$ $\mathrm{mg} / \mathrm{d}, 3=$ immunosuppressant drugs, regardless of steroid dose). ${ }^{13}$

\section{STATISTICAL ANALYSIS}

Outcome at three years follow up was divided into two groups: no increase in total damage score or increase in total damage score. Death was regarded as the worst outcome and therefore included in the latter group. Statistical analysis was carried out using logistic regression analysis (program logXact for Windows).

\section{Results}

One hundred and thirty three female and eight male patients with SLE (97 white subjects, 16 Afro-Caribbeans, 22 Asians, and 6 others) were included. Their age at inclusion was 41.1 (12.5) years and their disease duration 10.2 (6.3) years (range $0.1-32$ years). Table 1 shows their disease characteristics at the first assessment.

At the assessment three years after inclusion four patients were lost to the follow up because they had moved and seven patients had had the maximum damage as they had died during the follow up period. In 33/130 patients total SDI had increased and the mean damage score was now 1.5 (range 0-7) (table 1: damage scores at three years are given in square brackets). Table 2 shows the disease characteristics of the 40 patients with an increase in damage at three years and of those 97 with a stable damage score at follow up. Table 3 shows which variables predicted a negative outcome-that is, an increase in damage three years after the first assessment or death. Because 21 patients had failed to complete at least one section score of SF-20+, 116 patients were available for the analyses. Of those 116 patients, 33 showed increased damage at the end of the study (five of those 33 had died). The only variables at inclusion with an independent and significant contribution to predicting damage at three years were the total damage score $(\mathrm{OR}=1.46$; $95 \%$ CI 1.04 to 2.05 ) and the domain health 
Table 2 Disease characteristics at inclusion of the 40 patients with an increase in damage and of those 97 with a stable damage score at follow up and in brackets damage scores at follow up

\begin{tabular}{lll}
\hline SDI/BILAG; SF-20+; drug scores & $\begin{array}{l}\text { Patients with increase in } \\
\text { damage (median; range) }\end{array}$ & $\begin{array}{l}\text { Patients with stable } \\
\text { damage (median; range) }\end{array}$ \\
\hline Total damage & $1 ; 0-7(3 ; 1-7)$ & $0 ; 0-5(0 ; 0-5)$ \\
Neuropsychiatric damage & $0 ; 0-2(0 ; 0-3)$ & $0 ; 0-2(0 ; 0-2)$ \\
Renal damage & $0 ; 0-3(0 ; 0-3)$ & $0 ; 0-3(0 ; 0-3)$ \\
Musculoskeletal damage & $0 ; 0-2(0 ; 0-3)$ & $0 ; 0-3(0 ; 0-3)$ \\
BILAG total score & $4 ; 1-17$ & $4 ; 0-17$ \\
General BILAG & $1 ; 0-3$ & $1 ; 0-9$ \\
Mucocutaneous BILAG & $0 ; 0-3$ & $0 ; 0-9$ \\
Neurological BILAG & $0 ; 0-3$ & $0 ; 0-9$ \\
Musculoskeletal BILAG & $1 ; 0-9$ & $1 ; 0-9$ \\
Cardiovascular/respiratory BILAG & $0 ; 0-3$ & $0 ; 0-3$ \\
Vasculitis BILAG & $0 ; 0-3$ & $0 ; 0-3$ \\
Renal BILAG & $0 ; 0-3$ & $0 ; 0-3$ \\
Haematological BILAG & $0 ; 0-9$ & $0 ; 0-3$ \\
SF-20+: physical functioning & $50 ; 0-100$ & $67 ; 0-100$ \\
SF-20+: role functioning & $50 ; 0-100$ & $50 ; 0-100$ \\
SF-20+: social functioning & $60 ; 0-100$ & $80 ; 0-100$ \\
SF-20+: mental health & $68 ; 16-100$ & $68 ; 20-100$ \\
SF-20+: health perception & $30 ; 0-87$ & $47 ; 0-100$ \\
SF-20+: pain & $75 ; 0-100$ & $50 ; 0-100$ \\
SF-20+: fatigue & $70 ; 0-100$ & $70 ; 0-100$ \\
Drug score & $1 ; 0-3$ & $1 ; 0-3$ \\
\hline
\end{tabular}

Organ damage item only given if mean score at inclusion $\geqslant 0.1$ (and at three years $>0.12$ ). Lower scores always mean better health except for the SF-20+ scores marked with ${ }^{\star}$.

Table 3 Prediction of increase in total damage score at three years after inclusion or death $(n=116)$

\begin{tabular}{lll}
\hline Disease variable at inclusion & Odds ratio & $95 \%$ CI \\
\hline Age (years) & 1.006 & 0.96 to 1.05 \\
Disease duration (years) & 0.992 & 0.91 to 1.08 \\
Total damage score & 1.462 & 1.04 to $2.05^{\star}$ \\
Total BILAG score & 1.064 & 0.95 to 1.19 \\
Drug score & 1.051 & 0.74 to 1.5 \\
SF-20+: physical functioning & 0.991 & 0.97 to 1.01 \\
SF-20+: role functioning & 1.011 & 0.99 to 1.03 \\
SF-20+: social functioning & 1.005 & 0.99 to 1.02 \\
SF-20+: mental health & 1.016 & 0.99 to 1.04 \\
SF-20+: health perception & 0.961 & 0.93 to $0.99 \dagger$ \\
SF-20+: pain & 0.997 & 0.97 to 1.01 \\
SF-20+: fatigue & 0.998 & 0.98 to 1.02 \\
\hline${ }^{\star} \mathrm{p}=0.027$. & \\
$\dagger \mathrm{p}=0.009$. & &
\end{tabular}

perception of SF-20+ (OR=0.96; 95\% CI 0.93 to 0.99 ). There was no significant association between the total damage score and the general health perception score of SF-20+ (Spearman rank correlation coefficient $\left.\left(r_{s}\right)=-0.15\right)$ or the change of damage and disease duration $\left(r_{s}=0.13\right)$. Omitting the SF-20+ data, the logistic regression analysis of 137 patients showed the initial total damage score to be the only significant predictor of the outcome at three years $(\mathrm{OR}=1.401 ; 95 \%$ CI 1.08 to $1.82 ; \mathrm{p}=0.01)$. The logistic regression model used to calculate the outcome from the initial total damage and SF-20+: health perception score showed a positive predictive value (for the increase in damage at three years) of $59 \%$ and a negative predictive value (for stable damage score at three years) of $77 \%$ (specificity $92 \%$, sensitivity $30 \%$ ). If the initial total damage score only was used to predict the outcome at three years the positive predictive value (for the increase in damage at three years) was $42 \%$ and the negative predictive value (for stable damage score at three years) $72 \%$ (specificity $93 \%$, sensitivity $13 \%)$.

\section{Discussion}

In the present prospective study the prevalence of damage in at least one organ system in $56 \%$ of our patients with SLE is similar to that reported by Gorgos (59.8\%) in a retrospective analysis, as is the prevalence of damage in the three most commonly affected organ systems (neuropsychiatric, renal, and musculoskeletal), which ranges from 17 to $22 \%$ (12 to $25 \%$ reported by Gorgos). ${ }^{14}$ The mean damage score of our study of 1.2 is also similar to that of the Montreal cohort of 1.3, with a mean disease duration of 15 years. ${ }^{15}$ Our study group is also comparable with other SLE patient cohorts measured with the BILAG index. The percentage of SLE activity grade A (most active disease in an organ system) per number of assessments was $7.8 \%$ in our study, which is nearly identical with the rate of $8.2 \%$ reported previously. ${ }^{8}$ Although our study group is comparable with consecutive cohorts, it is not an inception cohort and might therefore have introduced a selection bias.

Logistic regression analysis (for a sample size of 116 and 137) showed the initial total damage score to be a significant predictor of damage three years later, which is in line with our previous work. ${ }^{5}$ Although being a focus of our interest, we could not analyse separately the outcome based on each organ damage score because the numbers of patients with a change in an organ damage score were too small for appropriate statistical analyses. This might be the aim of future studies with bigger patient cohorts.

Our hypothesis that in patients with SLE, as they have a systemic disease, the general health measure of SF-20+- that is, health perception (consisting of five questions), would best prognosticate outcome, was confirmed. However, this result has to be interpreted with caution and needs confirmation by other studies, as in $15 \%$ of the patients at least one section score of SF-20+ was missing and therefore only $82 \%$ of the cohort were available for the analysis $(n=116)$.

When calculating outcome using the logistic regression model, we correctly predicted in $93 \%$ of the patients with stable total damage score three years later that their damage would not increase. The predictive value for stable damage was $77 \%$. Unfortunately, adverse outcome, including increase in damage and death, was not predicted as acccurately (predictive value $59 \%$ ). Thus predicting adverse outcome with the intention to start a particular treatment for a patient or to prevent an unfavourable disease course remains of limited value and requires all the knowledge, experience, and intuition of the treating doctor. However, our study shows that based upon the present total damage score "no increase in damage three years later" was predicted correctly in the overwhelming majority of the patients with SLE, which allows the clinician to be reassuring in most cases.

Future prospective longitudinal studies of more than three years' duration and focused on larger and more homogeneous cohorts (in particular, inception cohorts) are needed to confirm and extend the results of our study. It would also be interesting to investigate the influence of comorbidity and the longitudinal 
sensitivity to change of the damage index in such studies, as neither was the subject of our study.

1 Stoll T, Gordon C, Seifert B, Richardson K, Malik J, Bacon PA, et al. Consistency and validity of patient administered assessment of quality of life by the MOS SF-36; its association with disease activity and damage in patients with systemic lupus erythematosus. J Rheumatol 1997;24:160814.

2 Hay EM. Systemic lupus erythematosus. Bailliere Clin Rheumatol 1995;9:447-70.

3 Gladman D, Ginzler E, Goldsmith C, Fortin P, Liang M, Urowitz M, et al. Systemic lupus international collaborative clinics: development of a damage index in systemic lupus clinics: development of a damage index in systemic lupus erythem $1820-1$.

4 Gladman D, Ginzler E, Goldsmith C, Fortin P, Liang M, Urowitz $M$, et al. The development and initial validation of the SLICC/ACR damage index for SLE. Arthritis Rheum 1996;39:363-9.

5 Stoll T, Seifert B, Isenberg DA. ACR/SLICC damage index is a useful predictor of severe outcome in SLE patients and an indicator of morbidity in different ethnic groups. $\mathrm{Br}$ Rheumatol 1996;35:248-54.

6 Stoll T, Stucki G, Malik J, Pyke S, Isenberg DA. Association of the systemic lupus international collaborating clinics/ American College of Rheumatology damage index with measures of disease activity and health status in patients with systemic lupus erythematosus. J Rheumatol 1997;24: with system-13.
7 Tan EM, Cohen AS, Fries JF, Masi AT, McShane DJ, Rothfield NF, et al. The 1982 revised criteria for the classification of systemic lupus erythematosus. Arthritis Rheum 1982;25:1271-7.

8 Hay EM, Bacon PA, Gordon C, Isenberg DA, Maddison P, Snaith ML, et al. The BILAG index: a reliable and valid instrument for measuring clinical disease activity in systemic lupus erythematosus. Q J Med 1993;86:447-58.

9 Stoll T, Stucki G, Malik J, Pyke S, Isenberg DA. Further validation of the BILAG disease activity index in patients with systemic lupus erythematosus. Ann Rheum Dis 1996; 55:756-60.

10 Stoll T, Stucki G, Gordon C, Isenberg DA. A damage index for lupus patients - where are we now? Lupus 1997;6:219 22 .

11 Stewart AL, Hays RD, Ware JE. The MOS short-form general health survey: reliability and validity in a patient population. Med Care 1988;26:724-35.

12 Wachtel T, Piette J, Mor V, Stein M, Fleishman J, Carpenter C. Quality of life in persons with human immunodeficiency virus infection: measurement by the medical outcom

13 Lacaille D, Clarke AE, Bloch DA, Danoff D, Esdaile JM The impact of disease activity, treatment and severity on short term costs of systemic lupus erythematosus. J Rheumatol 1994;21:448-53.

14 Gorgos L, Goldman D, Petri M. The ACR/SLICC damage index in systemic lupus erythematosus (SLE) [abstract]. Arthritis Rheum 1993;26(suppl 9):S68.

15 Fortin PR, Abrahamowicz M, Neville C, du Berger R, Fraenkel L, Clarke AE, et al. Impact of disease activity and cumulative damage on the health of lupus patients. Lupus 1998;7:101-7. 